\title{
Patients with acute exacerbations of COPD saw anxiety as a sign, rather than cause, of breathlessness
}

Bailey PH. The dyspnea-anxiety-dyspnea cycle-COPD patients' stories of breathlessness: "It's scary / when you can't breathe." Qual Health Res 2004; 14:760-78.

How do patients describe the relation between anxiety and the experience of dyspnoea (ie, perception of shortness of breath) during an acute exacerbation of chronic obstructive pulmonary disease (COPD)?

\section{DESIGN}

Focused ethnography with narrative analysis.

\section{SETTING}

2 hospitals in a mining community in northern Ontario, Canada.

\section{PATIENTS}

10 family-nurse units of patients who were admitted to hospital with acute exacerbation of COPD characterised by extreme dyspnoea; had $\geqslant 2$ previous acute exacerbations of COPD that required hospital admission; and had named family members as next of kin. The nurse-family units comprised 10 patients, 15 family caregivers (FCs), and 10 nurses.

\section{METHODS}

Indepth interviews were conducted during the patient's hospital stay. Questions were based on the chronic illness model of Strauss et al which defined acute COPD exacerbation as a medical crisis of a chronic illness. Narrative analysis was used to analyse 3 genres of the 503 stories identified from transcribed interviews: first-person eventspecific stories recreating a discrete moment in time; generic or habitual stories of the general course of events over time; and kernel stories suggesting untold first-person event-specific personal stories.

\section{MAIN FINDINGS}

The relation between acute dyspnoea and a patient's physical and emotional functioning was the most frequent topic of stories told by patients and FCs. Emotional vulnerability stories. Emotional vulnerability was expressed as anxiety experienced in anticipation of and during episodes of increasing or intractable breathlessness that patients could not avoid or manage. In stories where the relation between emotional function and breathlessness was unclear, participants talked of emotional dysfunction as a sign of intractable breathlessness. A complex and circular relation existed between breathlessness and anxiety: participants talked of emotional dysfunction as being the result of both chronic breathlessness and increased physical or emotional activity. Giving concrete expression to the experience of dyspnoea legitimised the illness and the help seeking behaviour of patients and FCs. Vulnerability was also understood in terms of patients' perceptions of lessened capacity for interacting with perceived threats in their environments. Sometimes, unusual emotional reactions to everyday situations (eg, arguing with a relative or being in a crowd) were understood as signs of the onset of unusual breathlessness; increasing dyspnoea then evoked other emotional reactions, which resulted in more breathlessness. Emotional vulnerability stories generally concluded with acknowledgement of patients' emotional disability, decreased activity, increased experience of dyspnoea, further emotional distress, and often, help seeking behaviour (eg, emergency admission to hospital).

Meaning of emotional vulnerability stories. Vulnerability stories helped to communicate that patients with COPD were emotionally vulnerable and that acute episodes of dyspnoea further exacerbated emotional dysfunction. Visibility function of vulnerability stories. Emotional dysfunction was presented as a visual expression of a

For correspondence: Dr P H Bailey, School of Nursing, Laurentian University, Sudbury, Ontario, Canada. pbailey@laurentian.ca

Sources of funding: Ontario Respiratory Care Society and Canadian Nurses' Research Society. patient's subjective experience of breathlessness or the sequellae of dyspnoea. Patients were seen as not being able to express common emotions without precipitating or exacerbating existing dyspnoea. Legitimisation function of emotional vulnerability stories. In their stories, patients measured the seriousness of dyspnoea by how they felt and what they were able to do. Patients and FCs understood emotional dysfunction as part of the experience of living with COPD, characterised by acute exacerbations. By giving dyspnoea a visible form, patients' complaints of an "invisible" experience (feeling short of breath) and their help seeking behaviours were legitimised; that is, breathlessness, concretely represented as emotional dysfunction and vulnerability, functioned to legitimise the experience of dyspnoea.

\section{CONCLUSIONS}

The vulnerability stories of patients with acute exacerbations of COPD and their family caregivers revealed an understanding of the dynamic relation between dyspnoea and emotional functioning, specifically anxiety. Anxiety was seen not as the underlying cause of distressing dyspnoea, but as a sign of longstanding or acute respiratory failure, a relation that could be described as the "dyspnoea-anxiety-dyspnoea cycle." Anxiety, in effect, was seen as a signal that patients were actually breathless.

Commentary

he findings of the qualitative study by Bailey reiterate the interrelation of emotion and breathing. As early as 1968, this interrelation in patients with COPD was described as an emotional straitjacket, where increased emotions lead to increased breathlessness or dyspnoea. ${ }^{1}$ Since these early discussions, both quantitative and qualitative investigations have attempted to clarify this relation and to treat the anxiety, with mixed results. ${ }^{2}$ The study by Bailey is consistent with the literature on anxiety and dyspnoea, but adds to it by including not only the perspectives of families, but also those of nurses. It is important to keep in mind that the emic nature of emotions and symptoms (ie, the meaning developed within the mind of an individual) makes it difficult to view the perceptions of individuals not experiencing an emotion or symptom with the same degree of appropriateness. ${ }^{3}$ Recent studies have found differences over time in the language used by individuals experiencing dyspnoea ${ }^{4}$ and differences in links between emotion and dyspnoea among physicians, nurses, and patients. ${ }^{5}$

The basic message for clinicians is that individuals with COPD commonly experience anxiety as a sign, and often not the cause, of breathlessness. The presence of anxiety during dyspnoea episodes is considered legitimate by patients and adds to the vulnerability experienced. Therefore, nurses should be careful not to "blame" patients by assuming that their dyspnoea is caused by their anxiety. Effective patient management should directly address patients' dyspnoea, rather than simply focusing on efforts to reduce their anxiety. Paula M Meek, RN, PhD, FAAN College of Nursing, University of New Mexico Albuquerque, New Mexico, USA

1 Dudley DL, Martin CJ, Holmes TH. J Psychosom Res 1968;11:325-39. 2 DeVito AJ. Heart Lung 1990;19:186-91.

3 "emic" The Concise Oxford Dictionary of Archaeology. Timothy Darvill. Oxford University Press, 2002. Oxford Reference Online. Oxford University Press. College of Santa Fe. Accessed 15 January 2005. hitp:// www. oxfordreference.com/views/

ENTRY. html? subview $=$ Main\&entry $=+102 . e 1353$

4 Michaels C, Meek PM. Heart Lung 2004:33:390-400.

5 Insel KC, Meek PM, Leventhal H. J Health Psychol 2005;10:147-62. 